\title{
A non-linear tracking control scheme for an under-actuated autonomous underwater robotic vehicle ${ }^{\dagger}$
}

\author{
Santhakumar Mohan ${ }^{1,2 *}$ and Asokan Thondiyath ${ }^{1}$ \\ ${ }^{1}$ Robotics Research Lab, Department of Engineering Design, Indian Institute of Technology Madras, Chennai 600-036, India \\ ${ }^{2}$ Ocean Robotics and Intelligence Lab, School of Mechanical, Aerospace and Systems Engineering, \\ Korean Advanced Institute of Science and Technology, Daejeon 305-701, Korea
}

(Manuscript Received June 17, 2011; Revised August 22, 2011; Accepted August 27, 2011)

\begin{abstract}
This paper proposes a model based trajectory tracking control scheme for under-actuated underwater robotic vehicles. The difficulty in stabilizing a non-linear system using smooth static state feedback law means that the design of a feedback controller for an under-actuated system is somewhat challenging. A necessary condition for the asymptotic stability of an under-actuated vehicle about a single equilibrium is that its gravitational field has nonzero elements corresponding to non-actuated dynamics. To overcome this condition, we propose a continuous time-varying control law based on the direct estimation of vehicle dynamic variables such as inertia, damping and Coriolis \& centripetal terms. This can work satisfactorily under commonly encountered uncertainties such as an ocean current and parameter variations. The proposed control law cancels the non-linearities in the vehicle dynamics by introducing non-linear elements in the input side. Knowledge of the bounds on uncertain terms is not required and it is conceptually simple and easy to implement. The controller parameter values are designed using the Taguchi robust design approach and the control law is verified analytically to be robust under uncertainties, including external disturbances and current. A comparison of the controller performance with that of a linear proportional-integral-derivative (PID) controller and sliding mode controller are also provided.
\end{abstract}

Keywords: Autonomous underwater robotic vehicle, under-actuated systems, non-linear control, ocean current, tracking control.

\section{Introduction}

Considering the importance of underwater technology in exploring the untapped resources beneath the sea, there seems to be a renewed interest in underwater robotics research, especially in developing advanced control strategies for Autonomous Vehicles. A summary of the recent developments in this area can be found in the literature [1, 2 \& 3]. Dynamics and Control of an Autonomous Underwater Vehicle (AUV) in a constrained environment poses significant challenges to designers. This

\footnotetext{
"Corresponding author. Tel.: +82-42-350-1590, Fax.: +82-42-350-1510.

E-mail address: santhakumar@kaist.ac.kr.

Copyright (C) KSOE 2011.
}

means that, coupled with the uncertainty of hydrodynamic parameters, the controller design becomes an extremely tough task. Additionally, most of the AUVs are under-actuated systems [1, 3, 4 \& 5], and this imposes non-integrable acceleration constraints on the vehicle where the classical control scheme fails. Under-actuated AUVs are considerably more difficult to control than fully actuated AUVs due to their non-linear and under-actuated nature. Until now, a globally uniformly asymptotically stabilizing control law for the general case has not been able to be created. Due to the structure of the system, it is impossible to find a time-invariant purestate feedback control scheme (locally or globally) that asymptotically stabilizes the origin [5]. There- 
fore, the exploration for a control law has centered on the area of discontinuous time-invariant control laws, continuous time-varying control laws or hybrids.

The problem of designing a stabilizing feedback controller for under-actuated systems is challenging since the system is not able to be stabilized by a smooth static state feedback law [6]. At the same time, a smooth feedback law can stabilize the position and velocity of a fully actuated vehicle (a vehicle where the control and configuration vector have the same dimension) asymptotically [1 \& 2]. Byrnes et al. have explained why under-actuated vehicles having zero gravitational fields are not asymptotically able to be stabilized around a single equilibrium [7]. On the other hand, Wichlund et al. have shown that a vehicle with gravitational and restoring terms in non-actuated dynamics is able to be stabilized around a single equilibrium point [8]. However, while this is a necessary condition, it is not sufficient to state that the vehicle is asymptotically able to be stabilized and this work mainly concentrated on ships (horizontal plane of the system focused).

The proposed control law addresses the closedloop asymptotic stability of the system and depends heavily on feedback linearization. However, it compensates all the non-linearities in the system by introducing non-linear elements in the input side, thus making the controller design more flexible [8, 9 \&10]. Unlike a traditional control, the proposed law can be thought of as the "instantaneous learning” of the vehicle dynamics. The control law requires the derivative of the state vector which can be derived from the onboard navigation sensors. The proposed control scheme exploits the inherent dynamics of the under-actuation system. The total number of states of the system is partitioned into actuated and non-actuated states and the control law is applied only to the actuated states. Using the Lyapunov direct method, an appropriate stabilizing tracking controller has been designed and its gain values are designed using the Taguchi optimization method. This work significantly differs from the previous work [11] in which it was assumed that there are four thrusters which can control the underactuated vehicle (yaw and pitch motions are directly controlled with thrusters) and the system matrices are also considered as diagonal (which makes the control independent of each other motion) matrices.
In the present work, the heave and sway (depended motions) of the vehicle motions are controlled with available control plane surfaces rather than thrusters and are controlled with the help of yaw and pitch motions respectively, while the system matrices also consist of off-diagonal elements. In other words, sway and pitch motions are controlled through the help of rudder plane (vertical) surfaces, and heave and pitch motions are controlled with the help of stern control (horizontal) surfaces. This task is rather complex compared to using controlling thrusters (direct control of yaw and pitch motions).

The remaining part of the paper is organized in the following manner: a brief discussion on the dynamic modeling of AUV is presented followed by the controller design details and its stability analysis. The simulation results and robustness of the proposed controller are presented for an experimental flat-fish AUV and a comparison of the results with that of a conventional PID controller and adaptive sliding mode controller is also provided.

\section{Modeling and simulation}

The dynamic model of an underwater vehicle is developed through the Newton-Euler formulation using the laws of conservation of linear and angular momentum. The equations of motion of such vehicles are highly non-linear [1] and are coupled due to hydrodynamic forces which act on the vehicle. Generally, the AUV model can be written with respect to either a body-fixed or an earth-fixed frame of reference.

The equations of motion of an underwater vehicle having six degrees of freedom with respect to a body-fixed frame of reference can be written as [1]:

$M \dot{v}+C(v) v+D(v) v+g(\eta)=\tau$

where,

$$
\begin{aligned}
& M=M_{R B}+M_{A}, \\
& C(v)=C_{R B}(v)+C_{A}(v), \\
& D(v)=D_{L}+D_{Q}|v|
\end{aligned}
$$

$M_{R B}$ and $C_{R B}(v)$ are the rigid body mass matrix and the Coriolis and centripetal matrix, respectively. $M_{A}$ and $C_{A}(v)$ are the added mass matrix and the added Coriolis and centripetal matrix respectively. $D_{L}$ and $D_{Q}$ are the linear and quadratic drag matrices, re- 
spectively. $g(\eta)$ is the resultant vector of gravity and buoyancy. $\tau=\left[\begin{array}{llllll}X & Y & Z & K & M & N\end{array}\right]^{\mathrm{T}}=B f$ is the resultant input vector of thruster, control plane forces and moments. $v=\left[\begin{array}{llllll}u & v & w & p & q & r\end{array}\right]^{\mathrm{T}}$ is the vector of linear and angular velocities in the vehicle coordinate frame. $\eta=\left[\begin{array}{llllll}x & y & z & \varphi & \theta & \psi\end{array}\right]^{\mathrm{T}}$ is the vector of absolute positions and Euler angles (roll, pitch and yaw). The relationship between linear and angular velocities in the vehicle frame to that in the absolute frame (refer Fig. 1) is given by:

$\dot{\eta}=J(\eta) v$

where, $J(\eta)$ is the kinematic transformation matrix and is given in the following form:

$J=\left[\begin{array}{cccccc}c \psi c \theta & -s \psi c \phi+c \psi s \theta s \phi & s \psi s \phi+c \psi c \theta s \phi & 0 & 0 & 0 \\ s \psi c \theta & c \psi c \phi+s \psi s \theta s \phi & -c \psi s \phi+s \psi s \theta c \phi & 0 & 0 & 0 \\ -s \theta & c \theta s \phi & c \theta c \phi & 0 & 0 & 0 \\ 0 & 0 & 0 & 1 & s \phi t \theta & c \phi t \theta \\ 0 & 0 & 0 & 0 & c \phi & -s \phi \\ 0 & 0 & 0 & 0 & s \phi / c \theta & c \phi / c \theta\end{array}\right]$

For the controller design and closed-loop stability analysis, it is preferred that the system is investigated with respect to the earth fixed frame of reference in order to maintain every state to a single reference frame. For this, the coordinate transformation $(\eta, v) \stackrel{\mu}{\rightarrow}(\eta, \dot{\eta})$ is performed using (3), which yields:

$\left(\begin{array}{l}\eta \\ \dot{\eta}\end{array}\right)=\left[\begin{array}{cc}I & 0 \\ 0 & J(\eta)\end{array}\right]\left(\begin{array}{l}\eta \\ v\end{array}\right)$

The coordinate transformation $\mu$ is a local diffeomorphism. This transformation is undefined for $\theta= \pm 90^{\circ}$ and to overcome this singularity, a quaternion approach must be considered. However, most of the AUVs are designed to operate at pitch angles well below $\pm 90^{\circ}$ and hence this limitation has no major significance here.

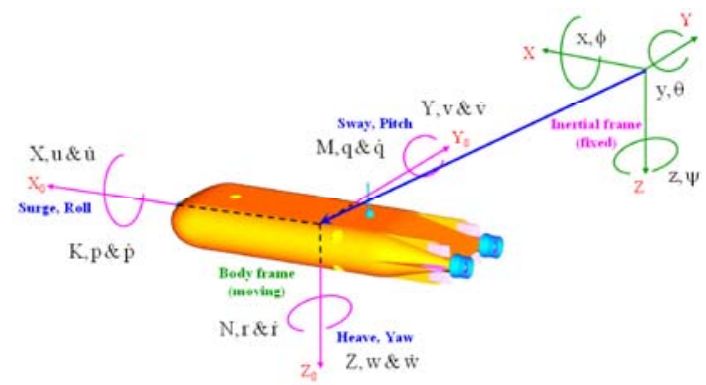

Fig. 1 Body-fixed frame and earth-fixed reference frame for AUV

The AUV dynamic model with respect to the earth fixed frame of reference becomes:

$$
M_{\eta}(\eta) \ddot{\eta}+C_{\eta}(v, \eta \eta) \dot{\eta}+D_{\eta}(v, \eta \eta) \dot{\eta}+g_{\eta}(\eta)=B_{\eta}(\eta) f
$$

where,

$$
\begin{aligned}
& M_{\eta}(\eta)=J(\eta)^{-T} M J(\eta)^{-1} \\
& C_{\eta}(v, \eta)=J(\eta)^{-T}\left(C(v)-M J(\eta)^{-1} \dot{J}(\eta)\right) J(\eta)^{-1} \\
& D_{\eta}(v, \eta)=J(\eta)^{-T} D(v) J(\eta)^{-1} \\
& g_{\eta}(\eta)=J(\eta)^{-T} g(\eta) \\
& B_{\eta}(\eta)=J(\eta)^{-T} B
\end{aligned}
$$

Here, [1]

$$
\begin{aligned}
& M_{\eta}(\eta)=M_{\eta}^{T}(\eta)>0, \forall \eta \in \mathfrak{R}^{6} \\
& s^{\mathrm{T}}\left[\dot{M}_{\eta}(\eta)-2 C_{\eta}(v, \eta)\right] s=0, \forall s \in \mathfrak{R}^{6}, v \in \mathfrak{R}^{6}, \eta \in \mathfrak{R}^{6} \\
& D_{\eta}(v, \eta)>0, \forall v \in \mathfrak{R}^{6}, \eta \in \mathfrak{R}^{6}
\end{aligned}
$$

\section{Non-linear Controller Design}

Our long term objective is to develop a real time, model-based, onboard non-linear motion controller for an under-actuated AUV, in order to improve the vehicle autonomy so as to enable it to carry out complex intervention tasks involving energy transfer between the AUV system and the environment. Such a controller can overcome the issues associated with the under actuation and the parameter variations such as buoyancy variation, uncertainties, disturbances and noises.

The first step in the development of such a real time controller is the development of a model-based, robust non-linear controller. In this paper, a nonlinear control technique is proposed and developed using the direct knowledge of vehicle dynamics. The robustness and performance of this control technique are demonstrated with the help of numerical simulations. A flat-fish shaped experimental AUV is considered here as the test platform. The details of controller development and simulation studies are presented below.

\subsection{Controller Development}

This section describes the design of the nonlinear tracking controller for the AUV to track a 
given reference trajectory using the model-based control law.

For an under-actuated system, the position vector $\eta$ can be partitioned to actuated and non-actuated states as

$$
\eta=\left[\begin{array}{ll}
\eta^{a} & \eta^{u}
\end{array}\right]^{\mathrm{T}}
$$

where, $\eta^{a}$ is the actuated states and $\eta^{u}$ is the nonactuated states of the AUV. The vehicle dynamics equation given in (6) can now be written as:

$$
\begin{aligned}
& {\left[\begin{array}{cc}
\tilde{M}_{\eta_{l l}} & \tilde{M}_{\eta_{12}} \\
\tilde{M}_{\eta_{2 l}} & \tilde{M}_{\eta_{22}}
\end{array}\right]\left[\begin{array}{l}
\ddot{\eta}^{a} \\
\ddot{\eta}^{u}
\end{array}\right]+\left[\begin{array}{ll}
\tilde{C}_{\eta^{\prime l}} & \tilde{C}_{\eta_{12}} \\
\tilde{C}_{\eta_{21}} & \tilde{C}_{\eta_{22}}
\end{array}\right]\left[\begin{array}{l}
\dot{\eta}^{a} \\
\dot{\eta}^{u}
\end{array}\right]} \\
& +\left[\begin{array}{cc}
\tilde{D}_{\eta_{l l}} & 0 \\
0 & \tilde{D}_{\eta_{22}}
\end{array}\right]\left[\begin{array}{l}
\dot{\eta}^{a} \\
\dot{\eta}^{u}
\end{array}\right]+\left[\begin{array}{c}
g_{\eta}^{a} \\
g_{\eta}^{u}
\end{array}\right]=\left[\begin{array}{c}
B_{\eta}^{a} \\
0
\end{array}\right] f
\end{aligned}
$$

This partition has some significance. Pettersen and Egeland [11 \& 12] considered a special case where $g_{\eta}^{u}$ corresponds to those directions that are not actuated. They showed that if $g_{\eta}^{u}$ contains zero elements, and there exists no continuous or discontinuous state feedback law, then the system is asymptotically stable. It is known that a necessary condition for a stable submersible body is that the centre of gravity (CG) should lie below the centre of buoyancy (CB). Ed: highlight - this is more fluent. For this, the AUV is trimmed (using a float) so that the CB is almost vertically above the CG and is nearly neutral buoyant. Here $g_{\eta}^{u}$ has non-zero components that represent self-restoring torque for the roll and pitch directions whilst $g_{n}^{a}$, with zero elements, represents the gravitational and buoyancy force vector corresponding to $\{u, v, w, r\}^{\mathrm{T}}$ directions that will need external control actions to stabilize [11, 12 \& 13]. With $g_{\eta}^{u}$ non-zero it may be possible to find continuous state feedback laws to stabilize the system as a whole. In addition to this, without loss of generality, we can assume that the damping terms of non-actuated states $\tilde{D}_{\eta_{22}}$ are sufficiently large than their inertia terms $\tilde{M}_{\eta_{22}}$ which means that the hydrodynamic restoring forces and torques are large enough to stabilize the nonactuated states (zero dynamics), which is a common property for AUVs [11]. This means that the robot can be exponentially stabilized by the actuated state controls alone. A flat-fish shaped AUV, as shown in Fig. 1, is considered here for modelling and analysis. This AUV has a length of $4.5 \mathrm{~m}$, width
$1.46 \mathrm{~m}$ and depth $0.73 \mathrm{~m}$. There are two propulsion thrusters for control of the longitudinal motion, four rudder planes and two stern planes for control of yaw and pitch respectively. The vehicle is underactuated due to the absence of lateral (sway) and vertical (heave) thrusters. Because of underactuation, sway, heave and roll motion cannot be controlled. However, sway and heave motions can be achieved through the help of rudder and stern planes during trajectory tracking but not during zero speed control. Vehicle inertial and hydrodynamic parameters are given in the annexure.

The actuated states alone are considered for the analysis and controller design, since the restoring effects allow the roll angle (un-actuated state) to stabilize itself effectively (refer Sec. 3.2). Hence, the system is analysed by neglecting the roll angle effects and the remaining states that are only considered for the controller design. Therefore, the reduced form of the equation of motion, which contains only actuated dynamics, is written as:

$M_{\eta}^{a} \ddot{\eta}^{a}+C_{\eta}^{a} \dot{\eta}^{a}+D_{\eta}^{a} \dot{\eta}^{a}+g_{\eta}^{a}=B_{\eta}^{a} f$

where, $\quad M_{\eta}^{a}=\tilde{M}_{\eta_{I I}}, C_{\eta}^{a}=\tilde{C}_{\eta_{I I}}$ and $D_{\eta}^{a}=\tilde{D}_{\eta_{I I}}$ are the mass/inertia, Coriolis/centripetal and hydrodynamic damping matrices associated with the actuated dynamics, respectively.

The dynamic model in (11) comprises non-linear functions of state variables and characterizes the behaviour of the vehicle in the actuated states. This feature of the dynamic model might imply that given any controller, the differential equation that models the control system in closed-loop should also be composed of non-linear functions of the corresponding state variables. This perception applies to most of the conventional control laws. Nevertheless, there exists a controller which is nonlinear in the state variables but which leads to a closed-loop control system described by linear differential equations. In the following section, we propose a model based controller (refer Proposition 1) which is capable of fulfilling the motion control objective globally with proper selection of its design parameters.

\section{Proposition 1}

Consider the system of which the governing equations are given by (11).

Let us define a positive definite Lyapunov function as: 


$$
V(\tilde{\eta}, \dot{\tilde{\eta}})=\frac{1}{2}[\dot{\tilde{\eta}}+\varepsilon \tilde{\eta}] \cdot[\dot{\tilde{\eta}}+\varepsilon \tilde{\eta}]+\frac{1}{2}\left[K_{P}+\varepsilon K_{D}-\varepsilon^{2} I\right] \tilde{\eta} \cdot \tilde{\eta}
$$

Choosing a control input of the form as given by (13) below:

$$
f=B_{\eta}^{a^{-1}}\left\{M_{\eta}^{a}\left(\ddot{\eta}_{d}^{a}+K_{D} \dot{\tilde{\eta}}+K_{P} \tilde{\eta}\right)+C_{\eta}^{a} \dot{\eta}^{a}+D_{\eta}^{a} \dot{\eta}^{a}+g_{\eta}^{a}\right\}
$$

will lead to the vehicle tracking position, orientation and velocity errors tending to zero asymptotically. i.e., the vehicle will follow the given desired trajectory.

Here, $K_{D}$ and $K_{P}$ are symmetric positive definite (SPD) design matrices, $\varepsilon$ is a positive constant, which satisfies $\lambda_{\min }\left\{K_{D}\right\}>\varepsilon>0, \lambda_{\min }$ is the minimum Eigen value of the matrix $K_{D} . \tilde{\eta}=\eta_{d}^{a}-\eta^{a}$ denotes the position and orientation errors of actuated states, $\quad \dot{\tilde{\eta}}=\dot{\eta}_{d}^{a}-\dot{\eta}^{a}$ denotes the velocity error, $\eta_{d}^{a}, \dot{\eta}_{d}^{a}$ and $\ddot{\eta}_{d}^{a}$ are the desired actuated states values of positions, velocities and accelerations respectively.

\section{Proof}

The above control input (u), given in (13) is substituted in the governing equation (11), which gives the closed-loop equation as:

$$
M_{\eta}^{a} \ddot{\eta}^{a}=M_{\eta}^{a}\left(\ddot{\eta}_{d}^{a}+K_{D} \dot{\tilde{\eta}}+K_{P} \tilde{\eta}\right)
$$

Since $M_{\eta}^{a}$ is a positive definite matrix, by property (8) and is invertible, (14) reduces to

$$
\ddot{\tilde{\eta}}+K_{D} \tilde{\dot{\eta}}+K_{P} \tilde{\eta}=0
$$

where, $\ddot{\tilde{\eta}}=\ddot{\eta}_{d}^{a}-\ddot{\eta}^{a}$ denotes the acceleration error. Equation (15) can be expressed in terms of the state vector $\left[\begin{array}{cc}\widetilde{\eta}^{\mathrm{T}} & \dot{\tilde{\eta}}^{\mathrm{T}}\end{array}\right]^{\mathrm{T}}$ as:

$$
\frac{\mathrm{d}}{\mathrm{dt}}\left[\begin{array}{c}
\tilde{\eta} \\
\dot{\tilde{\eta}}
\end{array}\right]=\left[\begin{array}{c}
\dot{\tilde{\eta}} \\
-K_{P} \tilde{\tilde{\eta}}-K_{D} \dot{\tilde{\eta}}
\end{array}\right]=\left[\begin{array}{cc}
0 & I \\
-K_{P} & -K_{D}
\end{array}\right]\left[\begin{array}{l}
\tilde{\eta} \\
\dot{\tilde{\eta}}
\end{array}\right]
$$

where, $I$ is the identity matrix of dimension $n$, which is equal to number of actuated states.

It is significant that the closed-loop equation (15) is represented by a linear autonomous differential equation, whose unique equilibrium point is given by $\left(\begin{array}{cc}\tilde{\eta}^{T} & \dot{\tilde{\eta}}^{T}\end{array}\right)^{\mathrm{T}}=0 \in \mathfrak{R}^{2 n}$. The uniqueness of the equilibrium is because the matrix $K_{P}$ is designed to be symmetric positive definite and therefore nonsingular. Since the closed-loop equation (15) is linear and autonomous, its solutions may be obtained in closed form and be used to conclude about the stability of the origin. However, the stability analysis of the origin as an equilibrium point of the closed-loop equation is analysed using Lyapunov's direct method [14].

Considering $\lambda_{\min }\left\{K_{D}\right\}>\varepsilon>0$, where $x \in \mathfrak{R}^{n}$ is any nonzero vector, we obtain

$$
\lambda_{\text {min }}\left\{K_{D}\right\} x . x>\varepsilon x . x
$$

Since $K_{D}$ is obtained by design, a symmetric positive definite matrix,

$$
\left(K_{D}-\varepsilon I\right) x . x>0, \quad \forall x \neq 0 \in \mathfrak{R}^{n}
$$

This means that the matrix $\left(K_{D}-\varepsilon l\right)$ is symmetric positive definite, i.e. $\left(K_{D}-\varepsilon I\right)>0$.

Considering all of the above, the matrix $K_{P}$ is symmetric positive definite and constant $\varepsilon$ also positive from the design; therefore,

$$
\left(K_{P}+\varepsilon K_{D}-\varepsilon^{2} I\right)>0
$$

Differentiating $V(\tilde{\eta}, \dot{\tilde{\eta}})$ (see (12)) with respect to time along the state trajectories, we obtain

$$
\dot{V}(\tilde{\eta}, \dot{\tilde{\eta}})=\dot{\tilde{\eta}} \cdot \ddot{\tilde{\eta}}+\left[K_{P}+\varepsilon K_{D}\right] \dot{\tilde{\eta}} \cdot \tilde{\eta}+\varepsilon \dot{\tilde{\eta}} \cdot \dot{\tilde{\eta}}+\varepsilon \dot{\tilde{\eta}} \cdot \tilde{\eta}
$$

Substituting from the closed-loop equation (15) in (20) and simplifying, we obtain

$$
\begin{aligned}
\dot{V}(\tilde{\eta}, \dot{\tilde{\eta}}) & =-\left[K_{D}-\varepsilon I\right] \dot{\tilde{\eta}} \cdot \dot{\tilde{\eta}}-\varepsilon K_{P} \tilde{\eta} \cdot \tilde{\eta} \\
& =-\left[\begin{array}{cc}
\varepsilon K_{P} & 0 \\
0 & K_{D}-\varepsilon I
\end{array}\right]\left[\begin{array}{c}
\tilde{\eta} \\
\dot{\tilde{\eta}}
\end{array}\right] \cdot\left[\begin{array}{c}
\tilde{\eta} \\
\dot{\tilde{\eta}}
\end{array}\right]
\end{aligned}
$$

Since the matrices $\left(K_{D}-\varepsilon l\right)$ and $K_{P}$ are symmetric positive definite matrices by design, the function $\dot{V}(\tilde{\eta}, \dot{\tilde{\eta}})$ in (21) is globally negative definite, i.e., $\dot{V}(\tilde{\eta}, \dot{\tilde{\eta}})<0$. From Lyapunov's stability theorem the equilibrium point of the closed-loop equation for the actuated states $\left(\begin{array}{cc}\tilde{\eta}^{T} & \dot{\tilde{\eta}}^{T}\end{array}\right)^{\mathrm{T}}=0 \in \mathfrak{R}^{2 n}$ is then globally uniformly asymptotically stable and therefore

$$
\begin{aligned}
& \lim _{t \rightarrow \infty} \dot{\tilde{\eta}}(t)=0 \\
& \lim _{t \rightarrow \infty} \tilde{\eta}(t)=0
\end{aligned}
$$


From (22), it can be observed that the tracking errors converge to zero asymptotically; therefore the vehicle follows the given desired trajectory.

\section{Note}

The proposed control law in (13) contains the term $K_{D} \dot{\tilde{\eta}}+K_{P} \tilde{\eta}$ which is of the PD type. However, this is pre-multiplied by the inertia matrix $M_{\eta}^{a}=M\left(\eta_{d}-\tilde{\eta}\right)$. Therefore this is not a linear controller as the case of PD, since the position and velocity gains are not constant but they depend explicitly on the position error. This may be clearly seen when expressing the proposed law given by (13) as:

$$
f=B_{\eta}^{a^{-1}}\left\{\begin{array}{l}
M\left(\eta_{d}-\tilde{\eta}\right) K_{D} \dot{\tilde{\eta}}+M\left(\eta_{d}-\tilde{\eta}\right) K_{P} \tilde{\eta} \\
+M_{\eta}^{a} \ddot{\eta}_{d}^{a}+C_{\eta}^{a} \dot{\eta}^{a}+D_{\eta}^{a} \dot{\eta}^{a}+g_{\eta}^{a}
\end{array}\right\}
$$

The control law in (23) makes use of the knowledge of the matrices $M_{\eta}^{a}, C_{\eta}^{a}, D_{\eta}^{a}$ and of the vector $g_{\eta}^{a}$ for calculating the control input $f$ and hence are referred as the model based control (MBC) law. The block diagram that corresponds to this controller is shown in Fig. 2.

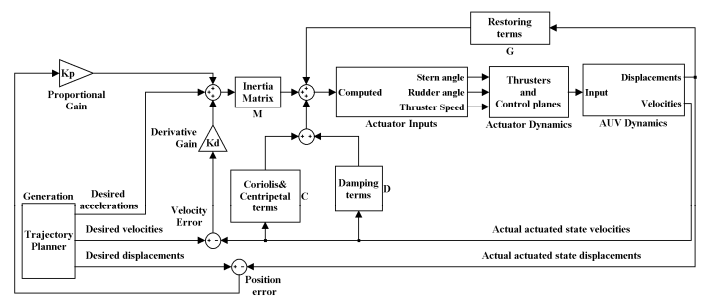

Fig. 2 Proposed vehicle controller structure

\subsection{Stability analysis of non-actuated state}

Here, the roll angle is the non-actuated state (i.e. there are no control inputs acted along this axis) and its equation of motion is given by:

$$
\begin{aligned}
& \left(I_{x}-K_{\dot{p}}\right) \dot{p}+\left(I_{z}-I_{y}\right) q r+\left(Y_{\dot{v}}-Z_{\dot{w}}\right) v w \\
& -Z_{\dot{q}} v q+\left(M_{\dot{q}}-N_{\dot{r}}\right) r q \\
& +Y_{\dot{r}} r w-K_{p} p-z_{B} B \cos \theta \sin \phi=0 \\
& \dot{\phi}=p
\end{aligned}
$$

Roll angle (non-actuated state) alone considered for the further analysis and all other states can be controlled with the available inputs. Therefore (24) can be linearized at its equilibrium point and it becomes:

$$
\begin{aligned}
& \left(I_{x}-K_{\dot{p}}\right) \dot{p}=K_{p} p+z_{B} B \phi, \\
& \text { where } \phi \text { is very small, } \therefore \sin \phi \approx \phi \\
& \dot{\phi}=p
\end{aligned}
$$

The linearized roll motion can be expressed in the following state space form:

$$
\begin{aligned}
& \left(\begin{array}{l}
\dot{p} \\
\dot{\phi}
\end{array}\right)=\left[\begin{array}{cc}
\frac{K_{p}}{I_{x}-K_{\dot{p}}} & \frac{z_{B} B}{I_{x}-K_{\dot{p}}} \\
1 & 0
\end{array}\right]\left(\begin{array}{l}
p \\
\phi
\end{array}\right) \\
& \left(\begin{array}{c}
\dot{p} \\
\dot{\phi}
\end{array}\right)=A\left(\begin{array}{c}
p \\
\phi
\end{array}\right)
\end{aligned}
$$

where,

$$
A=\left[\begin{array}{cc}
\frac{K_{p}}{I_{x}-K_{\dot{p}}} & \frac{z_{B} B}{I_{x}-K_{\dot{p}}} \\
1 & 0
\end{array}\right],
$$

$K_{p}$ and $K_{\dot{p}}$ are the hydrodynamic moment and added mass moment due to roll velocity and acceleration along the roll axis respectively. $I_{x}$ is the moment of inertia about $\mathrm{x}$ axis, $z_{B}$ is the $\mathrm{z}$ axis component of centre of buoyancy and B is the buoyancy value.

$$
\begin{aligned}
& K_{p}=-2050.3|U| \mathrm{kgm}^{2} / \mathrm{s}, K_{\dot{p}}=-645.85 \mathrm{kgm}^{2} / \mathrm{rad}, \\
& I_{x}=458.48 \mathrm{kgm}^{2}, \\
& z_{B}=-15 \mathrm{~mm} \text { and } B=14391.27 \mathrm{~N}
\end{aligned}
$$

(from annexure A.1 and values are dimensionalised)

$A=\left[\begin{array}{cc}-1.8566|U| & -0.1955 \\ 1 & 0\end{array}\right]$

Using Lyapunov's linearization method, we can demonstrate that the non-actuated state (roll motion) is asymptotically stable (i.e., if all the eigen values of the system matrix are strictly lie in the left-half complex plane then the equilibrium point is asymptotically stable). The system matrix (27) of the roll motion is dependent on the vehicle forward speed. Therefore the eigen values are calculated for different vehicle forward speeds, the forward speed varies 0 to $1.5 \mathrm{~m} / \mathrm{s}$ and the corresponding eigen values are plotted in the complex plane which is given in Fig. 3. This eigen values plot shows that the equilibrium point is asymptotically stable. However, if the vehicle forward speed is zero and the eigen values of the system only consist of imaginary parts, then the equilibrium point is marginally stable. 


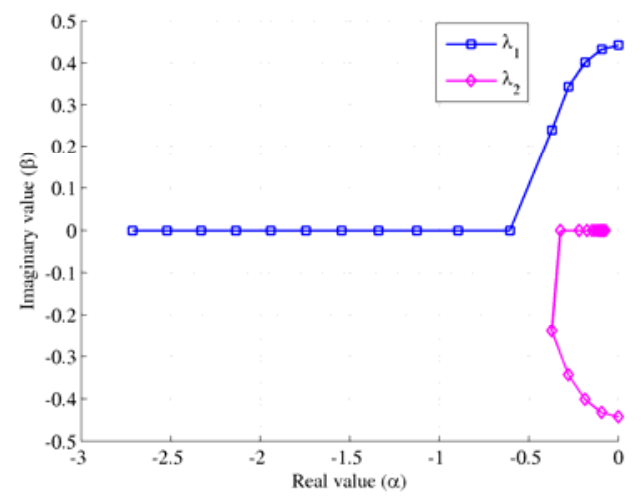

Fig. 3 Eigen values of zero dynamics (roll motion)

\section{Numerical Simulations}

The aim of this numerical simulation is to illustrate the performance of the proposed controller on an experimental autonomous underwater robot which is currently being developed at the Indian Institute of Technology Madras [15]. The vehicle is an under-actuated AUV; it has three control inputs (as mentioned as earlier), namely a set of thrusters to control surge motion (refer Fig. 1), and rudder surfaces and lateral moving surfaces (stern) to control steering and dive motion respectively [16 \& 17] Surge speed, pitch and yaw are considered here as controlled variables, while sway and heave are derived from yaw and pitch motions. Propulsion thrust, stern plane angle, and rudder angle are considered as the control variables (roll is not considered here as it cannot be controlled in this case).

This experimental vehicle is positive buoyant and has three control inputs, namely, propulsion thrusters for surge control and rudder and stern plane for attitude control. The robustness and the performance of the model based controller are compared with classical PID control and sliding mode control to illustrate the significance and performance improvement of the former [17]. The classical PID control input vector is defined for the system of which governing equation is in (11) and given by (28) as:

$$
f=B_{\eta}^{a^{-1}}\left\{K_{P} e+K_{D} \dot{e}+K_{I} \int e d t\right\}
$$

The sliding mode control (SMC) input vector is defined for the system of which governing equation is in (11) and given by (29) as [17-19]:

$$
f=-B_{\eta}^{a^{-1}}\left(K_{S} s+K \operatorname{sat}(s / \varphi)+\Lambda\right)
$$

where,

$s=\dot{e}+\lambda e$

$\Lambda=M_{\eta}^{a} \ddot{\eta}_{r}-C_{\eta}^{a} \dot{\eta}_{r}-D_{\eta}^{a} \dot{\eta}_{r}$

$\operatorname{sat}(s / \varphi)=\left\{\begin{array}{c}\operatorname{sgn}(s), i f|s / \varphi|>1 \\ s / \varphi, \text { otherwise }\end{array}\right\}$

$s$ is a sliding vector, $K_{S}$ and $K$ are sliding gains, $\lambda$ is a strict positive constant $(\lambda>0)$, taken to be the bandwidth of the system and $\varphi$ is the boundary layer thickness.

Simulation of the vehicle is performed using fourth order Runge - Kutta method with a fixed step size of $0.01 \mathrm{~s}$. The actuators are known to influence the overall vehicle dynamics, therefore the controller parameters (MBC, SMC and PID gains) are tuned by considering the actuator characteristics such as actuator response time (time constant, steady state time) and limits of actuators (saturation), etc using Taguchi robust design method [20] (please refer to [20] for detailed description of this tuning method). The design gain matrices of model based controller $\left(K_{P}\right.$ and $\left.K_{D}\right)$, PID controller $\left(K_{P}, K_{I}\right.$ and $\left.K_{D}\right)$ and sliding mode controller $\left(K_{s}\right.$ and $\left.K\right)$ are chosen diagonal. This means that the closed-loop systems given by (13), (28) and (29) are decoupled multivariable systems i.e., the dynamic behaviour of the errors of each state is governed by secondorder linear differential equations which are independent of each other.

The controller parameters used in the simulation (which are chosen by using Taguchi method as discussed in the annexure A. 2.) are as follows:

\section{Model based control (MBC):}

Proportional Gain $\left(K_{P}\right)=\operatorname{diag}(16,20,20)$ and Derivative Gain $\left(K_{D}\right)=\operatorname{diag}(4,2,10)$

\section{PID control:}

Proportional Gain $\left(K_{P}\right)=250 I_{3 x 3}$, Integral Gain $\left(K_{I}\right)=0.1 I_{3 \times 3}$ and Derivative Gain $\left(K_{D}\right)=500 I_{3 \times 3}$

\section{Sliding mode control (SMC):}

Bandwidth $(\lambda)=0.3$, boundary layer thickness $(\varphi)=15$, Sliding gain $\left(K_{s}\right)=120 I_{3 \times 3}$ and saturation gain $(K)=20 I_{3 \times 3}$ 
The same controller parameters (gains) are used for all the simulations throughout this paper.

The vehicle accelerates for the first 20 seconds, reaches a constant speed of $1.5 \mathrm{~m} / \mathrm{s}$ and decelerates in the last 20 seconds. The corresponding velocity and displacement plots are shown in Fig. 4 (b). The desired given 3D trajectory is depicted in Fig. 4. The proposed desired trajectory is chosen because it has four different motions i.e., heave up, heave down, sway in and sway out. Therefore vehicle behaviour in these directions can be understood easily. The comparative tracking controller for different control schemes are also given in the same figure (Fig. 4).

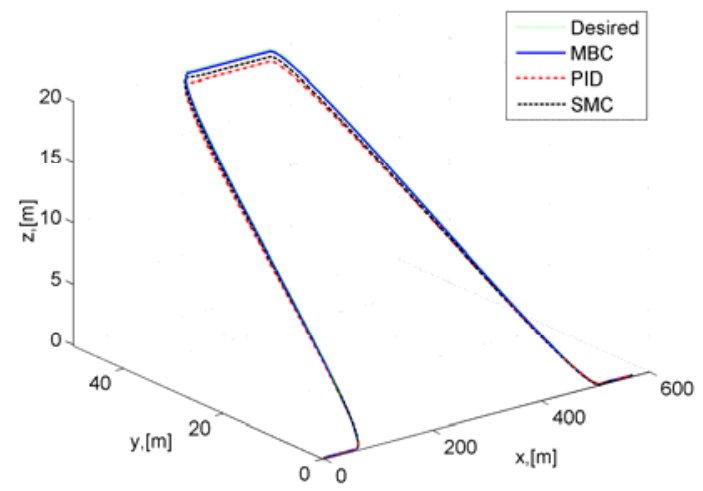

Fig. 4 Comparative trajectory tracking control results

Figure 5 shows the simulation results of position and attitude tracking errors during the commanded motion of the vehicle. It can be seen that the MBC significantly reduces the error in surge motion during the acceleration and deceleration phase, compared to that of PID and SMC control (refer Fig. 5). However, a small steady state error occurs during the constant velocity motion, which is well within the vehicle tolerance limits (the allowable position error values are $\pm 0.1 \mathrm{~m}$ and attitude error values are $\pm 0.2^{\circ}$ ). In case of the error going beyond the limits, it can be reduced by introducing an integral term in the proposed control law.

On the other hand, the controller responses are found to be in the opposite directions in the case of sway motion control (see sway error in Fig. 5). This behaviour is mainly due to the variations in the yaw response of the vehicle during PID and MBC con- trol (refer yaw error trajectory in Fig. 5), since sway and heave displacements are derived from the yaw and pitch motions. In the case of pitch control, it can be noted that the steady state error in heave is high. This is mainly due to the absence of an integral term in the controller. Integral term will reduce the steady state error to zero. This can be seen from heave tracking error trajectory in Fig. 5 (steady state error is present in MBC controller response but in PID controller, the error is converging to zero). The attitude error trajectory results show a definite improvement in the attitude errors in MBC control, however, the errors are not eliminated completely. This is mainly due to the time delay of control plane (actuator) response. The vehicle performance can be further improved by improving the actuator dynamics. The above results show that the tracking control of AUV can be significantly improved by introducing the proposed model based control strategy and is considerably more effective than the conventional PID control and sliding mode control.
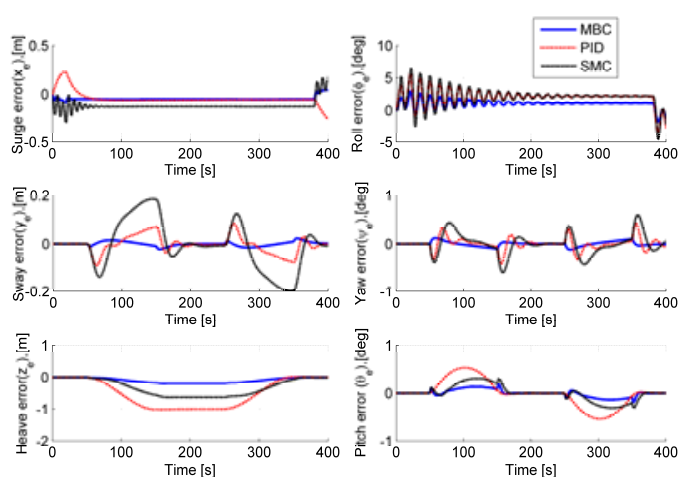

Fig. 5 Tracking errors for a given 3D trajectory

Although the performance of the proposed controller demonstrated successfully, a complex trajectory is also chosen and the controller performances are compared, while the complex desired trajectory with comparative results is given in Fig. 6 and tracking errors of this particular trajectory tracking is presented in Fig. 7. The comparative results show that the proposed controller performance is reasonable when compared to other controllers. Further analysis of the controller for robustness and disturbance rejection is presented below. 


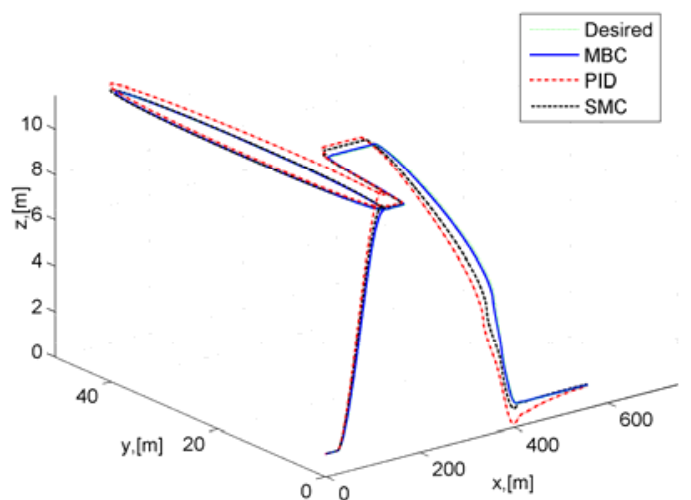

Fig. 6 Complex desired trajectory with comparative tracking control results
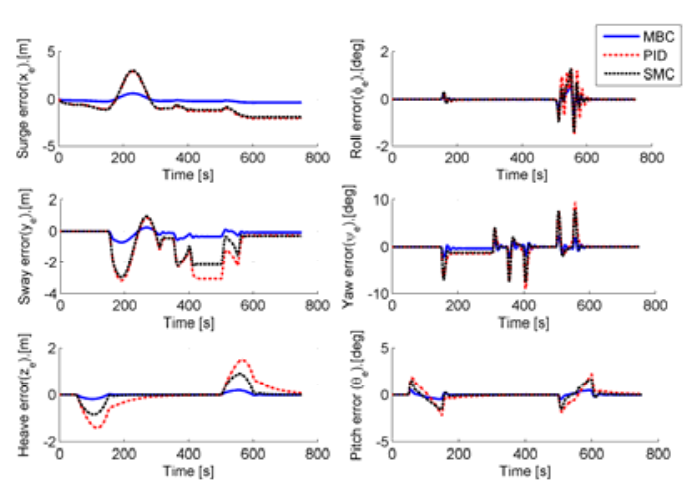

Fig. 7 Tracking errors for a given complex 3D trajectory

The above proposed control was verified through the help of limited closed-loop experiments in a towing tank. Due to availability and reliability of the sensors and subsystems, the trajectory is limited in the heave direction (only depth maneuver). The closed-loop trajectory of the vehicle was recorded using inertial navigation system and depth sensors, as the vehicle tracking based on the proposed control. The same condition was simulated using the dynamic model of the AUV and the results were compared. Figure 8 shows the comparison between experimental data and simulation results. It was found that the closed-loop response (depth trajectory) predicted by the dynamic simulation model agrees well with the experimental data. The tracking errors of simulated and experimental values of the prototype vehicle for the given depth trajectory is presented in Fig. 9.

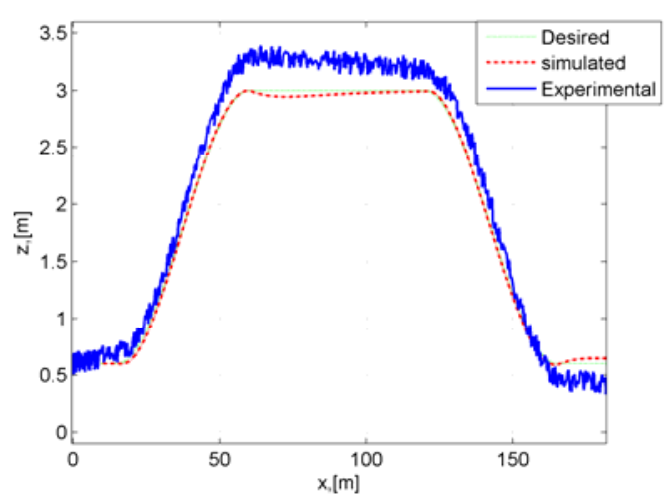

Fig. 8 Closed-loop response of the prototype vehicle for the depth trajectory
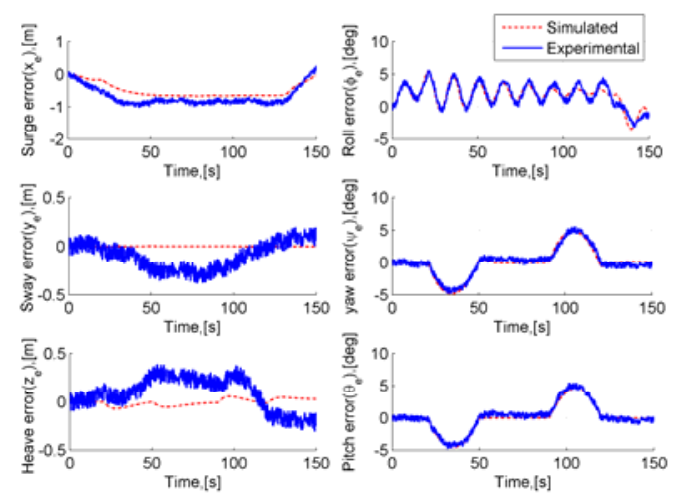

Fig. 9 Tracking errors for a given depth trajectory of a prototype vehicle

\section{Controller robustness and disturbance}

\section{rejection}

One important issue in underwater vehicle control is the initial error in position and orientation of the vehicle when the controller is switched on. Since most of the underwater vehicles are launched from the mother ship, there can be considerable amount of error in the position and orientation at the initial stage. Any new controller needs to be analysed for its ability to react to large initial errors. The model based controller proposed in this paper was subjected to a simulated initial error test to analyze its performance. The simulation results are presented in Fig. 10.

An initial error of $(1.2,1.0,0.8) \mathrm{m}$ in surge $(x)$, sway $(y)$, and heave $(z)$ positions, respectively and $\left(20^{\circ}, 12^{\circ}, 16^{\circ}\right)$ in roll $(\phi)$, pitch $(\theta)$ and yaw $(\psi)$ angles, respectively were simulated. The initial errors were found to converge to their desired states 
of zero over a short period of time, depending on the velocity of motion. The tracking errors of these conditions are converging to zero, as shown in the Fig. 10.
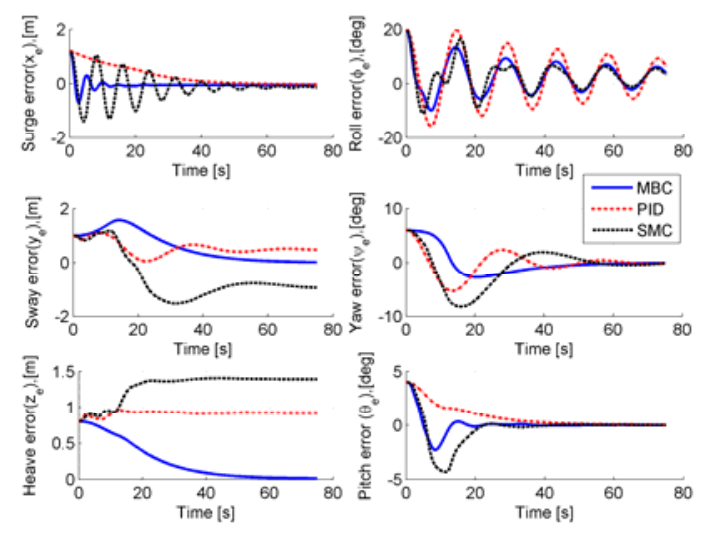

Fig. 10 Tracking errors with non-zero initial error

\subsection{Modeling of Underwater Current}

Underwater vehicles are frequently subjected to unidentified disturbances due to ocean currents. In this section, the effect of underwater current on vehicle motion is described and the performance of the proposed controller in the presence of underwater current is analysed. The underwater current model is developed in three dimensional space as described below.

Assuming that the fluid flow is irrotational, the earth fixed fluid flow velocity (velocity vector due to current) components can be related to the current velocity vector $V_{c}$ (Resultant velocity due to current) by defining the angle of attack $\alpha$ and side slip angle $\beta$, and describing the orientation of current velocity $V_{c}$ in the earth fixed frame. The ocean current detailed model [1] is given by (30) as:

$$
\left[\begin{array}{c}
u_{c}^{e} \\
v_{c}^{e} \\
w_{c}^{e}
\end{array}\right]=C_{y, \alpha} C_{z,-\beta}\left[\begin{array}{c}
V_{c} \\
0 \\
0
\end{array}\right]
$$

where, $C_{i, j}$ is the transformation matrix defined in [1], which denotes a rotation angle $j$ about $i^{-t h}$ axis, and $V_{c}$ is the average current velocity in the earth fixed frame. Expanding (30), we obtain the current velocity components in the earth fixed frame, namely:

$$
\begin{aligned}
& u_{c}^{e}=V_{c} \cos \alpha \cos \beta \\
& v_{c}^{e}=V_{c} \sin \beta \\
& w_{c}^{e}=V_{c} \sin \alpha \cos \beta
\end{aligned}
$$

The relative velocity of the vehicle $\left(v_{r}\right)$ with respect to the current velocity can be expressed as:

$v_{r}^{e}=\dot{\eta}-v_{c}^{e}$

Since the variation of current profile is slow, the time derivative of current velocity $\left(\dot{v}_{c}^{e}\right)$ is very small and it can be neglected. Therefore, the vehicle dynamics, represented by (11) can be rewritten as:

$M_{\eta}^{a} \ddot{\eta}^{a}+C_{\eta}^{a} v_{r}{ }^{e}+D_{\eta}^{a} v_{r}{ }^{e}+g_{\eta}^{a}=B_{\eta}^{a} f$

For this situation, the same proposed control law which is given in (13) is used without any further modification. Substituting (13) in (33) we obtain:

$$
\begin{aligned}
& M_{\eta}^{a} \ddot{\eta}^{a}+C_{\eta}^{a} v_{r}{ }^{e}+D_{\eta}^{a} v_{r}{ }^{e}+g_{\eta}^{a}=M_{\eta}^{a}\left(\ddot{\eta}_{d}^{a}+K_{D} \dot{\tilde{\eta}}+K_{P} \tilde{\eta}\right) \\
& +C_{\eta}^{a} \dot{\eta}^{a}+D_{\eta}^{a} \dot{\eta}^{a}+g_{\eta}^{a}
\end{aligned}
$$

The above equation shows that the controller does not require the direct knowledge of ocean current. The control law takes into account the ocean current without the need for the current measurement, since the current effects are directly replicating as the variations in positions and orientations and are observed by the navigation sensors during the vehicle motion. Using the dynamic model presented in (33) and the control law given in (13), the performance of the controller was tested for its ability to effectively control the adverse effects of underwater currents and external disturbances.

An average current velocity $\left(V_{c}\right)$ of $1 \mathrm{~m} / \mathrm{s}$ with an angle of attack $(\alpha)$ of $30^{\circ}$ and side slip angle $(\beta)$ of $30^{\circ}$ was assumed as well as external disturbances also considered as random (white) signals with a magnitude of $50 \mathrm{~N}$ and $50 \mathrm{Nm}$ in forces and moments. The simulation is carried out for the same desired trajectory (as described in the Sec. 4, refer Fig. 4) and the results are presented in Fig. 11. It can be observed from the results that the controller performs well in the presence of underwater currents. Except for a small steady state error, the controller is able to nullify the effects of current on tracking errors in surge, sway and heave. This would have been impossible with the use of PID control alone, as seen in Fig. 11. In the case of 
attitude control, we can see minor oscillations and resulting errors, especially in pitch and yaw control, when MBC is implemented (see Fig. 11). As the vehicle is being pushed out of the desired trajectory, the controller takes some time to respond and to return the vehicle to its trajectory. As the control forces exerted by the controller are a function of the velocity of the vehicle, the initial drift is high compared to the constant velocity phase of the trajectory. As the vehicle picks up speed, the error slowly settles down to a steady state value. These initial fluctuations and steady state errors can be further reduced by proper tuning of the controller.

Although this paper proposes a control method of an under-actuated flat-fish AUV, most of currently working AUVs are so-called 'Cruising type', which resemble a torpedo, and under actuated. It is therefore important to implement and improve control method for such vehicles. For this purpose, Jubilee AUV, the test-bed autonomous underwater vehicle (refer: Fig. 12 shows the first prototype of this vehicle, along with its experimental setup) is considered for the analysis. The proposed control is also developed for the Jubilee vehicle and its performance is demonstrated using the simulation results. Simulation results of Jubilee (torpedo-fish shaped) AUV is presented in Fig. 13. Here, for the simulation analysis, an average current velocity $\left(V_{c}\right)$ of 0.8 $\mathrm{m} / \mathrm{s}$ with an angle of attack ( $\alpha$ ) of $30^{\circ}$ and side slip angle ( $\beta$ ) of $30^{\circ}$ was assumed and external disturbances were also considered as random (white) signals with a magnitude of $5 \mathrm{~N}$ and $5 \mathrm{Nm}$ in forces and moments. The simulation is carried out for the same desired trajectory (as described in the Sec.4, refer Fig. 4). The simulation results from Fig. 13 show that the vehicle tracking errors are within the desired limits and performance of the controller is quite good. Therefore, the proposed controller can also be incorporated into cursing type vehicles.

The results presented above demonstrate the effectiveness of MBC in the presence of disturbance forces acting on the vehicle during a trajectory tracking mission.

\section{Note}

The proposed controller requires exact knowledge of the vehicle parameters, which is the limitation of this proposed controller. In this paper, experimen- tally calculated vehicle parameters are used and the uncertainties of the parameters are considered as disturbances. Since the controller is robust enough, the parameter variations can be compensated. It is also shown that such controller can be designed with the knowledge of inertia matrix alone where the Coriolis and centripetal, and damping effects can be negligible.
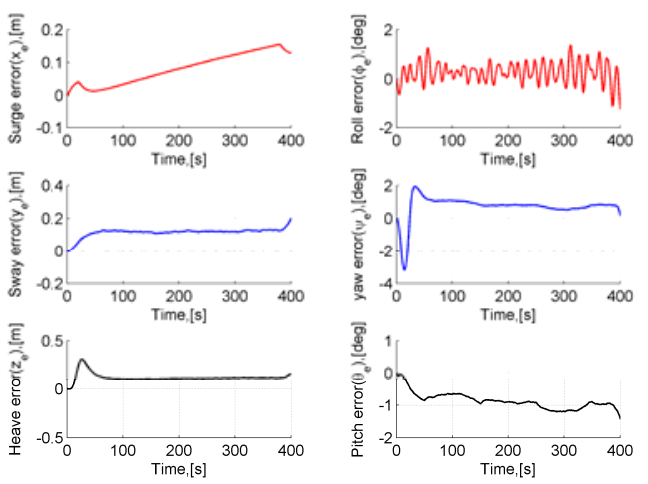

Fig. 11 Tracking errors in the presence of underwater current and random disturbances

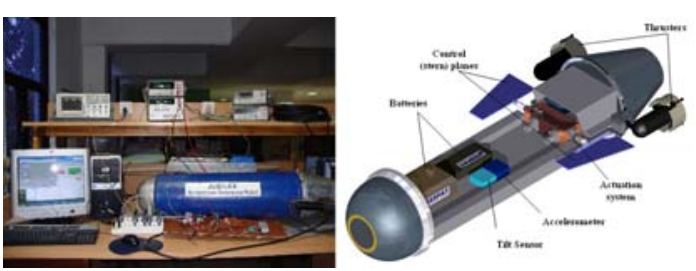

Fig. 12 Cursing type - experimental AUV (Jubilee) with its experimental setup
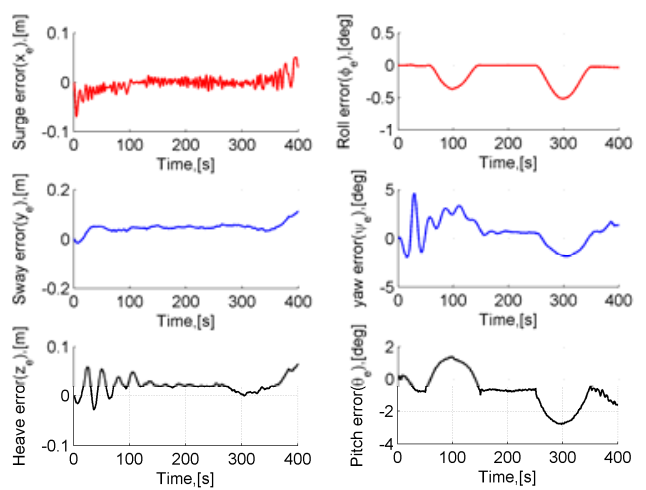

Fig. 13 Tracking errors of cruise type (torpedo shaped) vehicle in the presence of underwater current and random disturbances 


\section{Conclusions}

In this paper, a new robust trajectory control technique has been proposed for under-actuated underwater vehicles, based on vehicle dynamics. Simulation results show that the proposed controller is robust and can effectively track the given desired trajectory in the presence of uncertainties such as disturbances (underwater current). The simple structure, robustness and ease of computation of the proposed controller make it very attractive for real time implementation for underwater vehicle control. Compared to PID control, the proposed model based control is faster and more effective in reducing the tracking errors. Experimental validation of the proposed controller will be undertaken in the near future.

\section{Annexure}

\section{A.1 Experimental AUV details and specifications}

The experimental AUV is being developed in the Indian Institute of Technology Madras [15]. The vehicle shape is flat-fish and its specifications are as given in Tables 1-3. The hydrodynamic parameters are estimated using PMM experiments. These experiments were conducted at one of the $\mathrm{R}$ and $\mathrm{D}$ laboratories. Since the test results are used for real time experiments and controller development of the vehicle, they are reliable to the best of our knowledge.

Table 1 Experimental AUV parameters

\begin{tabular}{|c|c|}
\hline Parameters & Value in units \\
\hline Vehicle mass $(m)$ & 1462 kg \\
\hline Centre of gravity (CG) & {$[-22,0,0] \mathrm{mm}$} \\
\hline $\begin{array}{l}\text { Centre of buoyancy } \\
\text { (CB) }\end{array}$ & {$[-22,0,-15] \mathrm{mm}$} \\
\hline $\begin{array}{l}\text { Weight of the vehicle } \\
(W)\end{array}$ & $1462 * 9.81 \mathrm{~N}$ \\
\hline Buoyancy $(B)$ & $\begin{array}{l}1462(+10 \text { max. })^{*} \\
9.81 \mathrm{~N}\end{array}$ \\
\hline $\begin{array}{l}\text { Length of the vehicle } \\
(L)\end{array}$ & $4.5 \mathrm{~m}$ \\
\hline $\begin{array}{l}\text { Width of the vehicle } \\
\text { (b) }\end{array}$ & $1.46 \mathrm{~m}$ \\
\hline $\begin{array}{l}\text { Height of the vehicle } \\
\text { (h) }\end{array}$ & $0.73 \mathrm{~m}$ \\
\hline
\end{tabular}

\section{A.2 Controller Gain tuning using Taguchi's ro- bust design approach}

The robust parameter design is used to determine the levels of factors and to minimize the sensitivity to noise. That is, a parameter setting should be determined with the intention that the product response has minimum variation while its mean is close to the desired target. Taguchi's method is based on statistical and sensitivity analysis for determining the optimal setting of parameters to achieve robust performance.

In setting up a framework for robust design, the classification of the quantities at play in the design task is given below:

- Design variables (DV) are those quantities to be determined by the designer with the purpose of meeting performance specifications under given conditions.

- Design-environment parameters (DEP) are those quantities over which the designer has no control, and that define the conditions of the environment under which the designed object will operate.

- Performance functions (PF) are quantities used to represent the performance of the design in terms of design variables and designenvironment parameters.

The responses at each setting of parameters are treated as a measure that would be indicative of not only the mean of some quality characteristic, but also the variance of the same characteristic. The mean and the variance are combined into a single performance measure known as the signal-to-noise $(\mathrm{S} / \mathrm{N})$ ratio [21]. Taguchi classifies robust parameter design problems into different categories depending on the goal of the problem and for each category as follows:

Smaller is better: the target value of $y$, that is, quality variable is zero. In this situation, $\mathrm{S} / \mathrm{N}$ ratio is defined as follows:

$$
\text { S/N Ratio }=-10 \log \left(\frac{1}{n} \sum_{i=1}^{n} y_{i}^{2}\right)
$$

Larger is better: the target value of $y$, that is, quality variable is infinite and $\mathrm{S} / \mathrm{N}$ ratio is defined as follows:

$$
\text { S/N Ratio }=-10 \log \left(\frac{1}{n} \sum_{i=1}^{n} \frac{1}{y_{i}^{2}}\right)
$$


Nominal is best: the certain target value $(s)$ is given for $y$ value. In this situation $\mathrm{S} / \mathrm{N}$ ratio is defined as follows:

$$
\mathrm{S} / \mathrm{N} \text { Ratio }=-10 \log \left(\sum_{i=1}^{n} \frac{y_{i}^{2}}{s^{2}}\right)
$$

In this paper, the 'smaller is better' characteristic is used due to the requirement to minimize the tracking errors.

Taguchi's method uses an orthogonal array (OA) and analysis of mean to study the effects of parameters based on statistical analysis of experiments. An $\mathrm{OA}$ is a fractional factorial matrix which assures a balanced comparison of levels of any factor or interaction of factors. It is a matrix of numbers arranged in rows and columns where each row represents the level of the factors in each run, and each column represents a specific factor that can be changed from each run. The array is called orthogonal because all columns can be evaluated independently of each another.

Controller tuning involves the adjusting of the feedback controller parameters (gain values) to obtain a specified closed-loop response. It is very important that the controller gains obtained are robust even though system dynamics change and operating points vary. Here, the model based controller and PID controller gain values are designed (tuned) using this approach.

\section{Tuning of model based controller parameters}

In this section, the model based controller parameters are designed. The gain matrices $K_{P}$ and $K_{D}$ are in the following form, i.e., $K_{P}=\operatorname{diag}\left(K_{P x}\right.$, $\left.K_{\text {Ppitch }}, K_{\text {Pyaw }}\right)$ and $K_{D}=\operatorname{diag}\left(K_{D x}, K_{\text {Dpitch }}, K_{\text {Dyaw }}\right)$. where, $K_{P x}, K_{\text {Ppitch }}$ and $K_{\text {Pyaw }}$ are the proportional gains of surge, pitch and yaw respectively. Similarly $K_{D x}, K_{D p i t c h}$ and $K_{\text {Dyaw }}$ are the derivative gains of surge, pitch and yaw respectively. Three proportional gains and three derivative gains are to be designed. The parameter levels are chosen on the basis of actuator characteristics such as rise time and saturation limit. Here, five levels are chosen and their values are as follows: 4,8 , 12,16 and 20 are the five levels of the proportional gains and similarly 2, 4, 6, 8 and 10 are the five levels of the derivative gain values. The orthogonal array selection is carried out based on the number of factors and their levels. $\mathrm{L}_{25}\left(5^{6}\right)$ array has chosen for this analysis [21]. Conducting simulation experiments is based on the orthogonal array $\mathrm{L}_{25}\left(5^{6}\right)$ the sum of absolute error $(S A E)$ is calculated and recorded as given in the Table 4. With the help of these observations and the same procedure, $\mathrm{S} / \mathrm{N}$ ratio of each run is calculated and sum of the $\mathrm{S} / \mathrm{N}$ ratio of each factor and their each level values are calculated as shown in Table 5.

\section{Tuning of PID controller parameters}

The above procedure used for finding the robust gain values of PID control. The gain matrices $K_{P}$, $K_{D}$ and $K_{I}$ are in the following form, i.e., $K_{P}=k_{p} I$ $K_{D}=k_{d} I$ and $K_{I}=k_{i} I$, where, $k_{p}, k_{d}$ and $k_{i}$ are the proportional gain, derivative gain and integral gain of the PID control respectively. Here, three control parameters and three levels are chosen. Therefore orthogonal array $\mathrm{L}_{9}\left(3^{4}\right)$ is chosen for the analysis. Physical values of the each level are as follows: 150, 200 and 250 are the three levels of proportional gain values. Similarly 250, 500 and 750 are the three levels of derivative gain values. The integral gain level values are $0.05,0.1$ and 0.15 . With the help these values the simulation experiments have conducted as per $\mathrm{L}_{9}\left(3^{4}\right) \mathrm{OA}$, and the sum of absolute error $(S A E)$ is calculated and recorded as given in the Table 6. With the help of these observations and the same procedure, $\mathrm{S} / \mathrm{N}$ ratio of each run is calculated and sum of the $\mathrm{S} / \mathrm{N}$ ratio of each factor and each of their level values are calculated as shown in Table 7. From Table 7, it is found that the near optimum levels of controller parameters $k_{p}, k_{d}$ and $k_{i}$ are 3, 2 and 2 respectively, since these levels provide the maximum sum of the $\mathrm{S} / \mathrm{N}$ ratio value. The corresponding physical values of these particular levels are 250, 500 and 0.1 respectively. For further fine tuning of the controller parameters, the same procedure can be repeated.

Table 7 Sum of S/N ratio for different levels (PID)

\begin{tabular}{l|l|l|l}
\hline \hline \multirow{2}{*}{ Parameter } & \multicolumn{3}{c}{ Sum of S/N ratio for levels in dB } \\
& Level 1 & Level 2 & Level 3 \\
\hline $\boldsymbol{k}_{\boldsymbol{p}}$ & 33.44 & 38.41 & 41.76 \\
$\boldsymbol{k}_{\boldsymbol{d}}$ & 38.78 & 39 & 35.84 \\
$\boldsymbol{k}_{\boldsymbol{d}}$ & 37.88 & 38.14 & 37.59 \\
\hline
\end{tabular}


Table 2 Performance Specifications of Experimental AUV

\section{Vehicle details and performance characteristics}

\section{Vehicle shape}

Maximum vehicle speed

Depth of operation

Working sea condition

No. of Propulsion thrusters

No. of maneuvering / hovering thrusters

No. of control plane surfaces

Rudders (vertical surfaces)

Stern (horizontal rudder) control planes

Mode of operation

\section{Flat-fish type}

4 knots $\sim 6 \mathrm{~km} / \mathrm{hr}$

$400 \mathrm{~m}$ (max.)

Sea state 2

2

3

4 (combined operation)

2 (combined operation)

Propulsion thrusters and control plane surfaces only used.

No differential thrusters' action for any thrusters.

\section{Allowable design limits}

Position tracking errors

Attitude tracking errors

Drift

Water current speed

Allowable tracking back radius

Climb rate (ascend/descend)

Control surface limits

\section{$\pm 2 \mathrm{~m}$ \\ $\pm 5^{\circ}$}

$1 \% \mathrm{hr}$

1 knots (max.)

$200 \mathrm{~m}$ (max.)

$20^{\circ}$

in excess of $\pm 18^{\circ}$

Table 3 Non-dimensional hydrodynamic parameters

Added mass and hydrodynamic parameters

\begin{tabular}{l|l|l|l}
\hline$X_{\dot{u}}^{\prime}=-7.09 \times 10^{-3}$ & $Y_{\dot{v}}^{\prime}=-2.30 \times 10^{-2}$ & $Y_{\dot{r}}^{\prime}=4.86 \times 10^{-3}$ & $Z_{\dot{w}}^{\prime}=-1.54 \times 10^{-1}$ \\
$Z_{\dot{q}}^{\prime}=1.35 \times 10^{-3}$ & $K_{\dot{p}}^{\prime}=-7.0 \times 10^{-4}$ & $M_{\dot{q}}^{\prime}=-7.64 \times 10^{-3}$ & $N_{\dot{r}}^{\prime}=-9.30 \times 10^{-3}$ \\
$X_{u}^{\prime}=-1.34 \times 10^{-3}$ & $X_{v}^{\prime}=1.7 \times 10^{-3}$ & $X_{w}^{\prime}=-2.79 \times 10^{-3}$ & \\
$Y_{v}^{\prime}=-5.28 \times 10^{-2}$ & $Y_{r}^{\prime}=3.03 \times 10^{-2}$ & $Z_{w}^{\prime}=-1.42 \times 10^{-1}$ & $Z_{q}^{\prime}=-4.50 \times 10^{-2}$ \\
$K_{p}^{\prime}=-1.21 \times 10^{-4}$ & $M_{w}^{\prime}=6.81 \times 10^{-2}$ & $M_{q}^{\prime}=-3.16 \times 10^{-2}$ & $N_{v}^{\prime}=-8.16 \times 10^{-3}$ \\
$N_{r}^{\prime}=-5.82 \times 10^{-3}$ & $X_{u|| \mid}^{\prime}=-3.68 \times 10^{-3}$ & $X_{v|v|}^{\prime}=2.22 \times 10^{-3}$ & $X_{w|w|}^{\prime}=2.73 \times 10^{-3}$ \\
$Y_{v|v|}^{\prime}=-6.11 \times 10^{-2}$ & $Z_{w|w|}^{\prime}=-2.82 \times 10^{-1}$ & $M_{w|w|}^{\prime}=-8.36 \times 10^{-3}$ & $N_{v|v|}^{\prime}=2.20 \times 10^{-2}$ \\
$X_{\delta s}^{\prime}=-1.76 \times 10^{-3}$ & $X_{\delta r}^{\prime}=-7.61 \times 10^{-4}$ & $X_{\delta s|\delta s|}^{\prime}=-6.37 \times 10^{-2}$ & $X_{\delta r|\delta r|}^{\prime}=-1.86 \times 10^{-2}$ \\
$Y_{\delta r}^{\prime}=1.37 \times 10^{-3}$ & $Y_{\delta r|\delta r|}^{\prime}=6.19 \times 10^{-3}$ & $Z_{\delta s}^{\prime}=-7.26 \times 10^{-3}$ & $Z_{\delta s|\delta s|}^{\prime}=-8.36 \times 10^{-3}$ \\
$M_{\delta s}^{\prime}=-1.72 \times 10^{-3}$ & $M_{\delta s|\delta s|}^{\prime}=-4.81 \times 10^{-3}$ & $N_{\delta r}^{\prime}=-4.88 \times 10^{-3}$ & $N_{\delta r|\delta r|}^{\prime}=-2.63 \times 10^{-3}$ \\
\hline
\end{tabular}


Table 4 Design of experiments for model based control

\begin{tabular}{|c|c|c|c|c|c|c|c|c|}
\hline Exp & $K_{P x}$ & $K_{\text {Ppitch }}$ & $K_{\text {Pyaw }}$ & $K_{D x}$ & $K_{\text {Dpitch }}$ & $K_{\text {Dyaw }}$ & $S A E$ & S/N Ratio (dB) \\
\hline 1 & 1 & 1 & 1 & 1 & 1 & 1 & 188.11 & 4.51 \\
\hline 2 & 1 & 2 & 2 & 3 & 4 & 5 & 146.89 & 6.66 \\
\hline 3 & 1 & 3 & 3 & 4 & 5 & 2 & 134.82 & 7.41 \\
\hline 4 & 1 & 4 & 4 & 5 & 2 & 3 & 125.3 & 8.04 \\
\hline 5 & 1 & 5 & 5 & 2 & 3 & 4 & 119.53 & 8.45 \\
\hline 6 & 2 & 1 & 2 & 2 & 2 & 2 & 124.41 & 8.10 \\
\hline 7 & 2 & 2 & 3 & 5 & 1 & 4 & 95.508 & 10.40 \\
\hline 8 & 2 & 3 & 5 & 1 & 4 & 3 & 84.018 & 11.51 \\
\hline 9 & 2 & 4 & 1 & 4 & 3 & 5 & 111.2 & 9.08 \\
\hline 10 & 2 & 5 & 4 & 3 & 5 & 1 & 82.611 & 11.66 \\
\hline 11 & 3 & 1 & 3 & 3 & 3 & 3 & 102.36 & 9.80 \\
\hline 12 & 3 & 2 & 5 & 4 & 2 & 1 & 80.558 & 11.88 \\
\hline 13 & 3 & 3 & 4 & 2 & 1 & 5 & 68.692 & 13.26 \\
\hline 14 & 3 & 4 & 2 & 1 & 5 & 4 & 77.71 & 12.19 \\
\hline 15 & 3 & 5 & 1 & 5 & 4 & 2 & 96.126 & 10.34 \\
\hline 16 & 4 & 1 & 4 & 4 & 4 & 4 & 90.953 & 10.82 \\
\hline 17 & 4 & 2 & 1 & 2 & 5 & 3 & 102.84 & 9.76 \\
\hline 18 & 4 & 3 & 2 & 5 & 3 & 1 & 76.814 & 12.29 \\
\hline 19 & 4 & 4 & 5 & 3 & 1 & 2 & 59.7 & 14.48 \\
\hline 20 & 4 & 5 & 3 & 1 & 2 & 5 & 59.85 & 14.46 \\
\hline 21 & 5 & 1 & 5 & 5 & 5 & 5 & 84.277 & 11.49 \\
\hline 22 & 5 & 2 & 4 & 1 & 3 & 2 & 314 & 0.06 \\
\hline 23 & 5 & 3 & 1 & 3 & 2 & 4 & 89.117 & 11.00 \\
\hline 24 & 5 & 4 & 3 & 2 & 4 & 1 & 62.3 & 14.11 \\
\hline 25 & 5 & 5 & 2 & 4 & 1 & 3 & 63.624 & 13.93 \\
\hline
\end{tabular}

Table 5 Sum of S/N ratio for different levels (MBC)

\begin{tabular}{l|l|l|l|l|l|l}
\hline \hline \multirow{2}{*}{ Parameter } & \multicolumn{6}{l}{ Sum of S/N ratio for levels in dB } \\
& level 1 & level 2 & level 3 & level 4 & level 5 & Total \\
\hline $\boldsymbol{K}_{\boldsymbol{P X}}$ & 35.07 & 50.75 & 57.47 & 61.81 & 50.59 & 255.69 \\
$\boldsymbol{K}_{\text {Ppitch }}$ & 44.72 & 38.76 & 55.47 & 57.90 & 58.84 & 255.69 \\
$\boldsymbol{K}_{\text {Pyaw }}$ & 44.69 & 53.17 & 56.17 & 43.85 & 57.81 & 255.69 \\
$\boldsymbol{K}_{\boldsymbol{D} \boldsymbol{x}}$ & 42.74 & 53.68 & 53.60 & 53.11 & 52.56 & 255.69 \\
$\boldsymbol{K}_{\text {Dpitch }}$ & 56.58 & 53.48 & 39.68 & 53.45 & 52.50 & 255.69 \\
$\boldsymbol{K}_{\text {Dyaw }}$ & 54.45 & 40.39 & 53.04 & 52.86 & 54.94 & 255.69 \\
\hline
\end{tabular}

Table 6 Design of Experiments for PID control

\begin{tabular}{l|l|l|l|l|l|l}
\hline \hline Exp & $\boldsymbol{k}_{\boldsymbol{p}}$ & $\boldsymbol{k}_{\boldsymbol{d}}$ & $\boldsymbol{k}_{\boldsymbol{i}}$ & Error & $\boldsymbol{S} \boldsymbol{E}$ & S/N ratio (dB) \\
\hline 1 & 150 & 250 & 0.05 & 1 & 83.0708 & 11.61103 \\
2 & 150 & 500 & 0.1 & 2 & 84.4922 & 11.46367 \\
3 & 150 & 750 & 0.15 & 3 & 95.8676 & 10.36656 \\
4 & 200 & 250 & 0.1 & 3 & 69.2658 & 13.18962 \\
5 & 200 & 500 & 0.15 & 1 & 68.8422 & 13.24291 \\
6 & 200 & 750 & 0.05 & 2 & 79.6242 & 11.9791 \\
7 & 250 & 250 & 0.15 & 2 & 63.2466 & 13.97926 \\
8 & 250 & 500 & 0.05 & 3 & 60.9964 & 14.29392 \\
9 & 250 & 750 & 0.1 & 1 & 66.9156 & 13.48945 \\
\hline
\end{tabular}




\section{References}

[1] T.I. Fossen, Guidance and Control of Ocean Vehicles, Chichester, U.K: Wiley, (1994).

[2] J. Yuh, Design and control of autonomous underwater robots: a survey, Autonomous Robots, 8 (2000) 7-24.

[3] S. Zhao and J. Yuh, Experimental study on advanced underwater robot control, IEEE Transactions on Robotics, 21 (2005) 695703.

[4] C. Emanuele and C.M. Rinaldo, Conceptual design of an AUV equipped with a three degrees of freedom vectored thruster, Journal of Intelligent and Robotic Systems, 39 (2004) 365-391.

[5] K.Y. Pettersen and O. Egeland, Timevarying exponential stabilization of the position and attitude of an underactuated autonomous underwater vehicle, IEEE Transactions on Automatic Control, 44 (1999) 112-115.

[6] R.W. Brockett, Asymptotic stability and feedback stabilization, Differential Geometric Control Theory, Boston, (1983)181191.

[7] C. Byrnes and A. Isidori, On the attitude stabilization of rigid spacecraft, Automatica, 27 (1991) 87-95.

[8] K.Y. Wichlund, O.J. Sordalen and O. Egeland, Control of vehicles with second-order non-holonomic constraints: Underactuated vehicles, Proc. of the European Control Conference, Rome, Italy, (1995) 30863091.

[9] T.I. Fossen and O.E. Fjellstad, Robust adaptive control of underwater vehicle: A comparative study, Proc. of the IFAC Workshop on Control Applications in Marine Systems, Trondheim, Norway, (1995).

[10] T.I. Fossen, Marine Control Systems: Guidance, Navigation and Control of Ships, Rigs and Underwater Vehicles, Marine Cybernetics AS, (2002).

[11] K.Y. Pettersen and O. Egeland, Position and attitude control of an underactuated autonomous underwater vehicle, Proc. of the IEEE Conference on Decision \& Control, Japan, (1996) 987-991.

[12] K.Y. Pettersen and O. Egeland, Exponential stabilisation of an underactuated surface vessel, Proc. of the IEEE Conference on Decision \& Control, Japan, (1996) 967-972.

[13] T.H. Koh, M.W.S. Lau, G. Seet and E. Low, A control module scheme for an underactuated underwater robotic vehicle, Journal of Intelligent and Robotic Systems, 46 (2006) 43-58.

[14] J.J.E. Slotine and W. Li, Applied Nonlinear Control, Prentice-Hall, Englewood Cliffs, NJ, (1991).

[15] T. Asokan and M. Santhakumar, Mathematical Modelling and Simulation of Autonomous Underwater Vehicle, Technical Report, IIT Madras (2007).

[16] M. Santhakumar and T. Asokan, Coupled, non-linear control system design for autonomous underwater vehicle (AUV), Proc. of the IEEE International Conference on Control, Automation, Robotics and Vision (ICARCV 2008), Hanoi, Vietnam, (2008) 2309-2313.

[17] M. Santhakumar and T. Asokan, Investigations on the hybrid tracking control of an underactuated autonomous underwater robot, Advanced Robotics, 24 (2010) 15291556.

[18] R. Cristi, F.A. Papoulias and A.J. Healey, Adaptive sliding mode control of autonomous underwater vehicles in the dive plane, IEEE Journal of Oceanic Engineering, 15 (1990) 152-160.

[19] A.J. Healey and D. Lienard, Multivariable sliding mode control for autonomous diving and steering of unmanned underwater vehicles, IEEE Journal of Oceanic Engineering, 18 (1993) 327-339.

[20] M. Santhakumar and T. Asokan, Application of robust design techniques for underwater vehicle control, Proc. of the 8th ISOPE Ocean Mining Symposium (OMS2009), Chennai, India, (2009) 285-289.

[21] H.P. Sung, Robust Design and Analysis for Quality Engineering, Chapman and Hall, London (1996). 